\title{
SIMULATION OF SOUND STRUCTURE INTERACTIONS BY THE COUPLED FEM/BEM
}

\author{
ZAI-YOU YAN \\ College of Aerospace Engineering, Nanjing University of Aeronautics and Astronautics, P.R. China.
}

\begin{abstract}
Sound transmission through thin elastic shell with different fluids on the inside and outside is simulated using the in-house program based on the coupled finite element and boundary element method. The structure dynamics is simulated using the finite element method and the acoustic fields are simulated using the boundary element method. To avoid the non-uniqueness problem existing in the exterior acoustic boundary element method, Burton and Miller formulation is employed. The hyper-singular boundary integral is dealt with a regularization relationship. To validate this approach, a case with analytical solutions is simulated.
\end{abstract}

Keywords: boundary element method, Burton and Miller's formulation, finite element method, sound structure interaction

\section{INTRODUCTION}

Acoustic radiation and scattering from fluid-loaded elastic shells may be encountered in the aeronautical and naval industries as well as in underwater acoustics. It is well known that the presence of fluid modifies considerably the resonance characteristics of the structure. In the mean time, the propagation of sound in fluids is altered by the presence of the elastic structure, which causes serious noise problems. The radiated noise from a vibrating structure is important for underwater-related applications. The scattering of acoustic waves from such structures contains information relating to the geometry and composition of the structure, which makes it possible to identify the structure by the remote sensing. Therefore, it is of considerable interest to predict the acoustic fields both radiated and scattered by a submerged vibrating structure.

Acoustic radiation and scattering from submerged elastic structures have been studied by many researchers over the past seventy years. Numerous popular textbooks and monographs [1-3] have been devoted to this important subject. The overwhelming majority of investigations dealing with problems in structural acoustics have focused on the steady state response of the fluid-structure-coupled system. Therefore, it is natural that solutions have been carried out primarily in the frequency domain. Most of the problems addressed are about structures of revolution immersed in an unbounded acoustic fluid medium. The problem of sound radiation and scattering from shells of arbitrary shape with different fluids on both the inside and outside has been investigated very few. Analytical approaches to fluid-structure interaction problems are almost invariably concerned with spherical or infinite circular cylindrical shells subjected to axisymmetric excitations for which the classical method of separation of variables is available. For example, Junger [4] investigated the scattering of plane acoustic waves by air-filled, immersed in fluid elastic spherical and cylindrical shells. Goodman and Stern [5] investigated a submerged elastic spherical shell excited by plane acoustic waves using the exact theory of three-dimensional elasticity.

When analyzing the sound radiated or scattered by submerged elastic shells of more complicated shapes, it is almost indispensable to use numerical codes that can handle the complexity of the structure in question. For a complex structure subjected to known applied 
forces, the finite element method (FEM) has become an accepted, well-proven, and highly successful analysis tool. Therefore, for the sound/structure interaction model, it is almost consistent to formulate the structural dynamic equations via FEM techniques. When applying to the case of interior problems where the fluid is inside the structure, FEM also gives satisfactory solutions. However, in the case of exterior problem in which fluid occupies an unbounded domain, the FEM is inefficient. When FEM has been applied to unbounded exterior problems, the domain has to be truncated and radiating boundary conditions have to be enforced. In addition, they are limited by computer memory and runtime considerations as well. To deal with unbounded exterior acoustic field problems, the boundary element method (BEM) based on the utilization of the Helmholtz integral equation (HIE) has been the most popular numerical tool. Boundary integral formulations have long been recognized as an elegant and computationally economical method of modeling infinite fluid loading upon an elastic shell. The BEM method has several advantages over a FEM treatment of the acoustic problem, including a reduction of dimensionality of the problem by one, and an automatic satisfaction of the radiation condition. The elegance of this method is the mathematical simplicity of the resulting integral expressions. In the past few decades, numerical methods based on BEM had been employed to address the general acoustic radiation and scattering from elastic bodies.

One potential shortcoming of the classical BEM in acoustics is that the exterior boundary integral formulation shares the well-known difficulty of nonuniqueness of solution at certain characteristic frequencies. A well-known formulation to overcome the nonuniqueness problem is the method proposed by Burton and Miller [6].

To deal with the fluid-structure interaction problems, many researchers have developed FEM/BEM coupled schemes, using various boundary integral equations (BIEs) for the unbounded fluid domain together with a FEM to model the elastic target. The use of the same elements in both the FEM and BEM allows one to match the continuity at the fluid structure interface. Now, coupled FEM/BEM method is the most popular numerical tool for sound/structure interaction problems. Luke and Martin [7] gave a mathematical investigation about the coupled FEM and BEM method. They proved the existence, uniqueness theorems and so on. The first reference about coupled FEM/BEM method dated back to the late seventies. In 1977, Zienkiewicz et al. [8] proposed the coupled of the standard FEM to those derived from the BIEs. Subsequently, Wilton [9] formulated a coupled FEM/BEM system in terms of the acoustic variables. A comparison between the two different approaches for the coupled FEM/BEM, i.e. removal of the structural variables versus removal of the acoustic variables was made by Mathews [10]. Also using the submerged spherical shell, Mathews concluded that combining the equations in terms of the acoustic variables was both computational efficient and more accurate. Wu and Dandapani [11] used the coupled FEM and multi-domain boundary element method to model sound transmission through thin structures with different fluids on the inside and outside. As an example, they calculated the sound transmission through thin elastic spherical shell, which was submerged in water, filled with air, and subjected to a sound point source located at the center of the shell. Jeans and Mathews [12] presented a unique coupled FEM/ BEM method for the elastoacoustic analysis of fluid-filled thin shells. They concluded that except for problems having a significant density difference between the internal and external acoustic fields, for example, air and water, their formulation was suitable.

This paper numerically investigates the sound transmission through thin elastic shell with different fluids on both the inside and outside using numerical method. To deal with such kind of structural-acoustics coupled problems, a numerical model based on the coupled finite/ boundary element method (FEM/BEM) is developed, in which the elastic target is modeled 
using the finite element method and the acoustic equations are solved using the boundary element method. Finally, the finite element model and the boundary models are completely coupled through the continuity conditions on the fluid structure interfaces. Example with analytical solution is simulated to verify the developed method.

\section{FINITE ELEMENT MODEL FOR SHELLS}

The general shell element employed here had been described in Ref. [13]. The elements are eight-nodded isoparametric quadrilateral elements. Strains normal to the midsurface are neglected, and lines originally normal to the midsurface are constrained to remain straight during deformation. The effect of transverse shear deformation has been included. Therefore, the rotation of the midsurface normal vector is an independent variable.

According to Hamilton's principle, the finite element governing equation [10] for the dynamic fluid-structure interaction system is given by

$$
\left[-\omega^{2} M-i \omega C+K\right]\{U\}=\left\{F_{I}\right\}+\left\{F_{A}\right\}
$$

where $M, K$ and $C$ are the mass, stiffness and damping matrices, respectively. $U$ denotes the displacement and $\omega$ is the circular frequency. $F_{A}$ represents the known applied excitation forces and $F_{I}$ represents the interaction forces generated by the acoustic fluid acting on the fluid-structure interaction surfaces. The vector of interaction force can be defined through the structure coupled matrix $L_{s}\left(L_{s}^{i}\right.$ and $\left.L_{s}^{e}\right)$ and the nodal acoustic pressures $\left\{\varphi^{i}\right\}$ and $\left\{\varphi^{e}\right\}$, that is

$$
\left\{F_{I}\right\}=L_{s}^{i}\left\{\varphi^{i}\right\}-L_{s}^{e}\left\{\varphi^{e}\right\}
$$

where $\left\{\varphi^{i}\right\}$ and $\left\{\varphi^{e}\right\}$ are the interior and exterior surface acoustic pressures, respectively. $L_{s}^{i}$ and $L_{s}^{e}$ are the structure coupled matrices on the interior surface and exterior surface. The coupled matrix of element is defined as

$$
L_{s}=\iint_{S}[N]_{f}^{T}\{\vec{n}\}[N] d S
$$

where $[N]_{f}^{T}$ is the shape function matrix about displacement in the finite element method. $[N]$ is the shape function matrix about acoustic pressure in the boundary element method and $\{\vec{n}\}$ is the vector of the direction cosines of normal vector. Detail discussion of the coupled matrix will be presented in Ref. [14].

Previous to describe the general shell element, eight-node isoparametric element displayed in Fig. 1 will be introduced first. Isoparametric elements are formulated using an natural coordinate system $\xi \eta$, which is defined by element geometry and not by the element orientation in the global coordinate system $x y$. Coordinates $\xi \eta$ (and $\zeta$, if the element is three-dimensional) are attached to the element and are scaled so that sides of a quadrilateral element shown in Fig. 1 are defined by $\xi=-1, \xi=1, \eta=-1$ and $\eta=1$, as shown in Fig. 2 . Shape functions [14] for quadratic eight-node isoparametric curvilinear quadrilateral elements are given as follows:

$$
\begin{aligned}
& N_{1}=-\frac{1}{4}(1-\xi)(1-\eta)(1+\xi+\eta), N_{2}=\frac{1}{2}\left(1-\xi^{2}\right)(1-\eta) \\
& N_{3}=\frac{1}{4}(1+\xi)(1-\eta)(\xi-\eta-1), N_{4}=\frac{1}{2}(1-\xi)\left(1-\eta^{2}\right)
\end{aligned}
$$




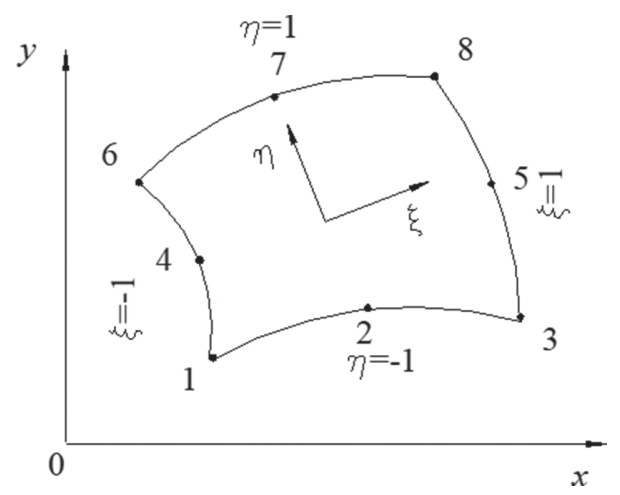

Figure 1: Curvilinear quadrilateral element.

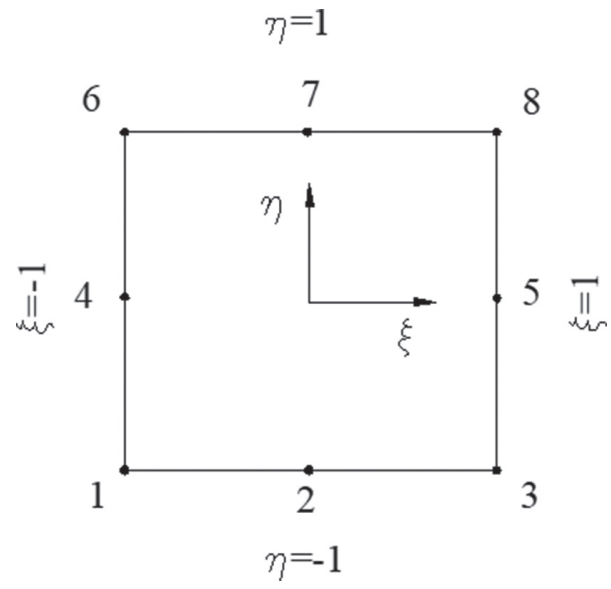

Figure 2: Parent element.

$$
\begin{gathered}
N_{5}=\frac{1}{2}(1+\xi)\left(1-\eta^{2}\right), N_{6}=\frac{1}{4}(1-\xi)(1+\eta)(-1-\xi+\eta) \\
N_{7}=\frac{1}{2}\left(1-\xi^{2}\right)(1+\eta), N_{8}=\frac{1}{4}(1+\xi)(1+\eta)(\xi+\eta-1)
\end{gathered}
$$

Therefore, the shape function matrix $[N]$ can be expressed as follows

$$
[N]=\left[\begin{array}{llllllll}
N_{1} & N_{2} & N_{3} & N_{4} & N_{5} & N_{6} & N_{7} & N_{8}
\end{array}\right]
$$

For thin shell, the solid element can be reduced to an eight-node isoparametric surface element, as shown in Fig. 3. Note that the mid-surface normal needs not to be kept normal during deformation; it may take angles of other 90 degrees with the deformed mid-surface. In this way, the ability to model transverse deformation is retained. Consider a local curvilinear coordinate system $o \xi \eta \zeta$ on each element. Let $\xi$ and $\eta$ be curvilinear coordinates on the middle surface. $\zeta=1$ represents upper surface $S_{u}$ and $\zeta=-1$ represents lower surface $S_{l}$. 


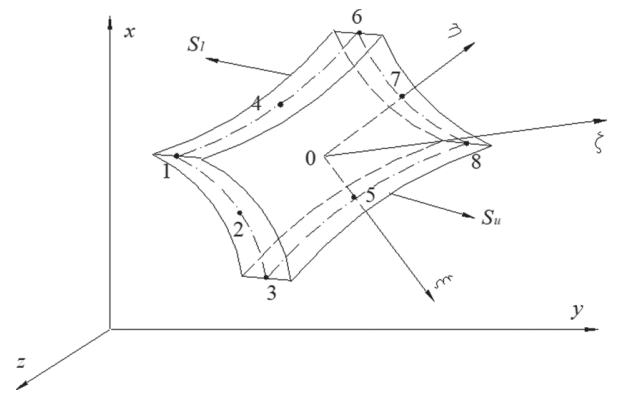

Figure 3: Eight-node isoparametric mid-surface element.

A unit normal vector on middle surface through node $i$ is given by

$$
\vec{V}_{3 i}=\left\{\begin{array}{c}
l_{3 i} \\
m_{3 i} \\
n_{3 i}
\end{array}\right\}=\frac{1}{h_{i}}\left(\left\{\begin{array}{l}
x_{i} \\
y_{i} \\
z_{i}
\end{array}\right\}_{u}-\left\{\begin{array}{l}
x_{i} \\
y_{i} \\
z_{i}
\end{array}\right\}_{l}\right)
$$

where, $l_{3 i}, m_{3 i}$ and $n_{3 i}$ are the direction cosines of $\vec{V}_{3 i}$ in the global coordinate system and $h_{i}$ is the thickness of the shell structure.

Then, the global coordinates of arbitrary point in the solid element (see Fig. 3) can be obtained using the shape functions eqn (4) as

$$
\left\{\begin{array}{l}
x \\
y \\
z
\end{array}\right\}=\sum_{i=1}^{8} N_{i}(\xi, \eta)\left(\left\{\begin{array}{l}
x_{i} \\
y_{i} \\
z_{i}
\end{array}\right\}+\frac{h_{i}}{2} \zeta \vec{V}_{3 i}\right)
$$

Suppose the mid-surface normal line rotates about two different axes, which are perpendicular to each other and parallel to the mid-surface. Let $\vec{V}_{1 i}$ and $\vec{V}_{2 i}$ are the unit vectors of these two axes respectively.

The corresponding rotation angles of normal vector $\vec{V}_{3 i}$ around the axes $\vec{V}_{1 i}$ and $\vec{V}_{2 i}$ are $\beta_{i}$ and $\alpha_{i}$. Then the rotation vector can be expressed by

$$
\vec{\omega}_{i}=\beta_{i} \vec{V}_{1 i}+\alpha_{i} \vec{V}_{2 i}
$$

Consequently, the displacements of arbitrary point on the normal vector $\vec{V}_{3 i}$ can be expressed using the mid-surface nodal displacements $u_{i}, v_{i}, w_{i}$ and rotation vector $\vec{\omega}_{i}$ of mid-surface normal line. By the shape function interpolation, the displacements of arbitrary point in the solid element are

$$
\left\{\begin{array}{l}
u \\
v \\
w
\end{array}\right\}=\sum_{i=1}^{8} N_{i}(\xi, \eta)\left(\left\{\begin{array}{l}
u_{i} \\
v_{i} \\
w_{i}
\end{array}\right\}+\zeta\left[\phi_{i}\right]\left\{\begin{array}{l}
\alpha_{i} \\
\beta_{i}
\end{array}\right\}\right)
$$

where $\phi_{i}$ is defined as follows

$$
\left[\phi_{i}\right]=\left[\begin{array}{ll}
\phi_{11 i} & \phi_{12 i} \\
\phi_{21 i} & \phi_{22 i} \\
\phi_{31 i} & \phi_{32 i}
\end{array}\right]=\frac{h_{i}}{2}\left[\begin{array}{cc}
l_{1 i} & -l_{2 i} \\
m_{1 i} & -m_{2 i} \\
n_{1 i} & -n_{2 i}
\end{array}\right]=\frac{h_{i}}{2}\left[\begin{array}{ll}
\vec{V}_{1 i} & -\vec{V}_{2 i}
\end{array}\right]
$$


Equation (8) can be expressed in standard form as

$$
\left\{\begin{array}{c}
u \\
v \\
w
\end{array}\right\}=[N]_{f}\left\{\begin{array}{c}
\left\{\delta_{1}\right\} \\
\left\{\delta_{2}\right\} \\
\vdots \\
\left\{\delta_{8}\right\}
\end{array}\right\}=\sum_{i=1}^{8}\left[N_{i}\right]_{f}\left\{\delta_{i}\right\}
$$

where $\left\{\delta_{i}\right\}=\left[\begin{array}{lllll}u_{i} & v_{i} & w_{i} & \alpha_{i} & \beta_{i}\end{array}\right]^{T}$ is the vector for degrees of freedom at node $i$. The matrix $[N]_{f}$ is defined as the shape function matrix for displacements.

$$
[N]_{f}=\left[\begin{array}{ccccccccccc}
N_{1} & 0 & 0 & N_{1} \zeta \phi_{111} & N_{1} \zeta \phi_{121} & \ldots & N_{8} & 0 & 0 & N_{8} \zeta \phi_{118} & N_{8} \zeta \phi_{128} \\
0 & N_{1} & 0 & N_{1} \zeta \phi_{211} & N_{1} \zeta \phi_{221} & \ldots & 0 & N_{8} & 0 & N_{8} \zeta \phi_{218} & N_{8} \zeta \phi_{228} \\
0 & 0 & N_{1} & N_{1} \zeta \phi_{311} & N_{1} \zeta \phi_{321} & \ldots & 0 & 0 & N_{8} & N_{8} \zeta \phi_{318} & N_{8} \zeta \phi_{328}
\end{array}\right]_{3 \times 40}
$$

The element stiffness matrix can be written in block form. That is each element stiffness matrix, $[k]_{40 \times 40}$, can be partitioned into sixty-four $\left[k_{i j}\right]_{5 \times 5}$ element sub-matrices. These submatrices will be calculated in terms of the following integral equation [14]

$$
\left[k_{i j}\right]=\int_{-1}^{1} \int_{-1}^{1} \int_{-1}^{1}\left[B_{i}\right]^{T}[D]\left[B_{j}\right]|J| d \xi d \eta d \zeta
$$

The expressions for the matrices $B$ and $D$ can be found in the reference [14]. $J$ represents the Jacobian matrix.

The element consistent mass matrix can be expressed by using the shape functions for displacements as

$$
[m]=\int_{-1}^{1} \int_{-1}^{1} \int_{-1}^{1}[N]_{f}^{T} \rho[N]_{f}|J| d \xi d \eta d \zeta
$$

The structure damping matrix $C$ is implemented numerically through the use of the following hysteric relationship [12].

$$
C=\gamma K
$$

Here $\gamma$ is assumed as the structural loss factor and $K$ is the structure stiffness matrix. In general, $\gamma$ is chosen to be 0.01 .

\section{BOUNDARY ELEMENT MODEL FOR ACOUSTICS}

An elastic thin shell is defined on the closed surface $S$. The shell submerged in an infinite fluid with density $\rho^{e}$ in the exterior domain $E$ and contains a fluid with density $\rho^{i}$ in the interior domain $D$. The fluids on the inside and outside are assumed to be inviscid and compressible. The surface of the shell is assumed smooth. The normal vector is defined to point into the exterior domain, as shown in Fig. 4.

A harmonic time dependency term of $e^{i \omega t}$ is assumed and the wave number $k$ can be defined as

$$
k=\omega / c
$$

where $\omega$ is the circular frequency and $c$ is the sound velocity 


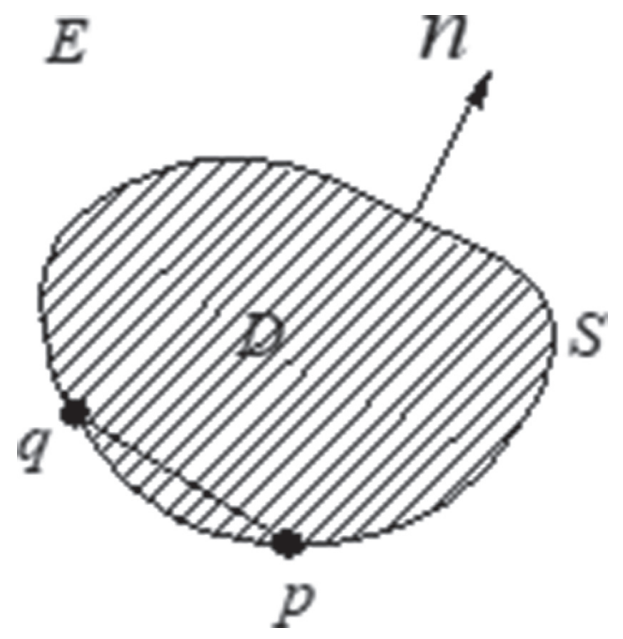

Figure 4: Notation for submerged fluid-filled shell.

Assuming there are a point sound source in the interior domain and a plane acoustic wave in the exterior domain. Then, the acoustic pressure $\varphi^{e}$ in the exterior domain satisfies the homogeneous reduced wave equation

$$
\left(\nabla^{2}+k^{2}\right) \varphi^{e}(p)=0
$$

The point sound source with strength $\Omega$ is located at the point $p_{0}$ in the interior domain. Then, the incident acoustic pressure $\varphi_{I}^{i}$ produced by the point sound source can be expressed as

$$
\varphi_{I}^{i}(r)=\frac{\Omega}{4 \pi} \frac{e^{-i k r}}{r}
$$

The acoustic problems are defined using the reduced wave equations and boundary conditions on the surfaces of the shell. The partial derivatives of the exterior surface acoustic pressure $\varphi^{e}$ and interior surface acoustic pressure $\varphi^{i}$ with the surface normal displacements $u_{n}^{e}$ and $u_{n}^{i}$ satisfy the following relations.

$$
\begin{aligned}
& \frac{\partial \varphi^{e}}{\partial n}=\omega^{2} \rho^{e} u_{n}^{e} \\
& \frac{\partial \varphi^{i}}{\partial n}=\omega^{2} \rho^{i} u_{n}^{i}
\end{aligned}
$$

Besides the above boundary continuity conditions, the pressure difference between the exterior acoustic pressure $\varphi^{e}$ and the incident plane acoustic wave pressure $\varphi_{I}^{e}$ is subjected to the Sommerfeld radiation condition.

The Helmholtz integral equation for exterior acoustic pressure $\varphi^{e}$ is

$$
c^{e}(p) \varphi^{e}(p)=\iint_{S}\left[\varphi^{e}(q) \frac{\partial G(p, q)}{\partial n_{q}}-G(p, q) \frac{\partial \varphi^{e}(q)}{\partial n_{q}}\right] d S_{q}+\varphi_{I}^{e}(p)
$$


And the interior acoustic pressure $\varphi^{i}$ can be expressed in the form of boundary integral equation as

$$
c^{i}(p) \varphi^{i}(p)=\iint_{S}\left[\varphi^{i}(q) \frac{\partial G(p, q)}{\partial n_{q}}-G(p, q) \frac{\partial \varphi^{i}(q)}{\partial n_{q}}\right] d S_{q}-\varphi_{I}^{i}(p)
$$

where $c^{e}(p)$ and $c^{i}(p)$ are the dimensionless solid angles on the exterior and interior surfaces respectively.

In operator notations, the surface Helmholtz integral equations can be given as,

$$
\begin{gathered}
{\left[-\frac{1}{2} I+M_{k}\right] \varphi^{e}=L_{k} \frac{\partial \varphi^{e}}{\partial n}-I \varphi_{I}^{e}} \\
{\left[\frac{1}{2} I+M_{k}\right] \varphi^{i}=L_{k} \frac{\partial \varphi^{i}}{\partial n}-I \varphi_{I}^{i}}
\end{gathered}
$$

where the integral operators $M_{k}$ and $L_{k}$ are defined as

$$
M_{k} \mu=\iint_{S} \mu \frac{\partial G}{\partial n} d S, L_{k} \mu=\iint_{S} \mu G d S
$$

Burton and Miller [6] had shown that a combination of the surface Helmholtz integral equation (22) and its differentiation will provide a unique solution for the entire frequency range. It can be represented in operator notation as

$$
\left[-\frac{1}{2} I+M_{k}+\alpha N_{k}\right] \varphi^{e}=\left[L_{k}+\alpha\left[\frac{1}{2} I+M_{k}^{T}\right]\right] \frac{\partial \varphi^{e}}{\partial n}-I \varphi_{I}^{e}-\alpha I \frac{\partial \varphi_{I}^{e}}{\partial n}
$$

where $\alpha$, the coupling constant, is chosen to be strictly complex. Generally, $\alpha$ is taken to be $-i / k . i$ is an imaginary unit. The integral operators $N_{k}$ and $M_{k}^{T}$ are defined as

$$
N_{k} \mu=\iint_{S} \mu(q) \frac{\partial^{2} G(p, q)}{\partial n_{q} \partial n_{p}} d S_{q}, M_{k}^{T} \mu=\iint_{S} \mu(q) \frac{\partial G(p, q)}{\partial n_{p}} d S_{q}
$$

The hypersingular integral in the operator $N_{k}$ is dealt with the regularization relationship developed in Refs. $[14,15]$. Since this regularization relationship reduces the hyersingular integral to weakly singulars, $C^{0}$ element is enough to compute the hyersingular integral.

\section{THE COUPLED FEM/BEM MODEL}

There are two kinds of continuity boundary conditions on the fluid structure interfaces. One is the kinetic boundary condition and the other is the dynamic boundary condition.

\subsection{Kinetic continuity boundary condition}

The partial derivatives of the surface acoustic pressures $\varphi^{e}$ and $\varphi^{i}$ with the surface normal displacements $u_{n}^{e}$ and $u_{n}^{i}$ satisfy eqns (18) and (19). The nodal values of normal displacements $\left\{u_{n}\right\}$ and displacements $\{U\}$ have the following relationship

$$
\left\{u_{n}\right\}=Q\{U\}
$$


where $Q$ is defined as the kinetic coupled matrix. It can be explicitly expressed as

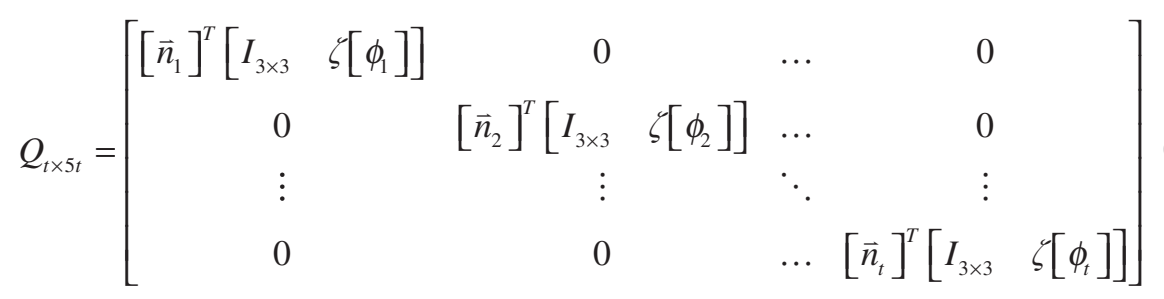

where $t$ is the total number of node. Matrix $Q$ is not a square one. It can be stored using one-dimensional compacted storage.

$$
Q= \begin{cases}Q^{e} & \zeta=1 \\ Q^{i} & \zeta=-1\end{cases}
$$

Then, the kinetic continuity boundary condition can be expressed as

$$
\begin{aligned}
\frac{\partial \varphi^{e}}{\partial n} & =\omega^{2} \rho^{e} Q^{e}\{U\} \\
\frac{\partial \varphi^{i}}{\partial n} & =\omega^{2} \rho^{i} Q^{i}\{U\}
\end{aligned}
$$

\subsection{Dynamic boundary condition}

For problems of sound transmission through a fluid-filled, submerged shell structure, the coupled nodal forces $\left\{F_{I}\right\}$ satisfy the eqn (2). The element-coupled matrix $L$ can be partitioned into 64 sub-matrices $L_{i j}$ of 5 by 1 .

$$
L_{i j}=\iint_{S}\left[N_{i}\right]_{f}^{T}[\vec{n}] N_{j} d S
$$

where

$$
\left[N_{i}\right]_{f}^{T}[\vec{n}]\left[N_{j}\right]=\left[\begin{array}{c}
l_{3} N_{i} N_{j} \\
m_{3} N_{i} N_{j} \\
n_{3} N_{i} N_{j} \\
a_{i} \zeta N_{i} N_{j} \\
b_{i} \zeta N_{i} N_{j}
\end{array}\right]_{5 \times 1}, i, j=1,2, \cdots, 8
$$

and

$$
\begin{aligned}
& a_{j}=l_{3} \phi_{11 j}+m_{3} \phi_{21 j}+n_{3} \phi_{31 j}, j=1,2, \cdots, 8 \\
& b_{j}=l_{3} \phi_{12 j}+m_{3} \phi_{22 j}+n_{3} \phi_{32 j}, j=1,2, \cdots, 8
\end{aligned}
$$

The structure-coupled matrix $L_{s}$ is not a square one. Assuming the total node number is $t$, because there are five degrees of freedom at each node, the dimension of structure matrix $L_{s}$ is $5 t \times t$. The matrices $L^{e}$ and $L^{i}$ with the matrix $L$ satisfy the following relation 


$$
L= \begin{cases}L^{e} & \zeta=1 \\ L^{i} & \zeta=-1\end{cases}
$$

Then, combing the eqns (1), (2), (21) and (24), sound transmission through thin elastic shell with different fluids on both the inside and outside can be solved.

\section{NUMERICAL EXAMPLE}

In order to validate the correctness of the present coupled FEM/BEM method, a fluid-filled submerged elastic spherical shell excited by a point sound source located at the center is analyzed. In this example, the fluid mediums inside and outside the spherical shell are air and sea water, respectively. The spherical shell is discretized 96 surface element. The external radius of the spherical shell is $1.01 \mathrm{~m}$ and the internal radius is $1.0 \mathrm{~m}$. Therefore, the relative thickness of the spherical shell is $1 \%$. It is a thin shell. The density of the spherical shell is $\rho=7.81 \times 10^{3} \mathrm{~kg} / \mathrm{m}^{3}$. The elasticity modulus is $E=2.07 \times 10^{11} \mathrm{~Pa}$ and Poisson's ratio is $\mu=0.3$. The air density is $\rho^{a}=1.21 \mathrm{~kg} / \mathrm{m}^{3}$ and sound velocity in air is $c^{a}=346 \mathrm{~m} / \mathrm{s}$. While, the sea water density is $\rho^{w}=1030 \mathrm{~kg} / \mathrm{m}^{3}$ and sound velocity in sea water is $c^{w}=1500 \mathrm{~m} / \mathrm{s}$.

The frequency range is from 50 to $2000 \mathrm{~Hz}$. Figure 5 shows the comparison of the exterior surface acoustic pressure amplitude obtained using the coupled FEM/BEM and the corresponding analytical solutions [11]. The numerical results agree with the corresponding analytical solutions throughout the frequency range. Figure 6 shows the

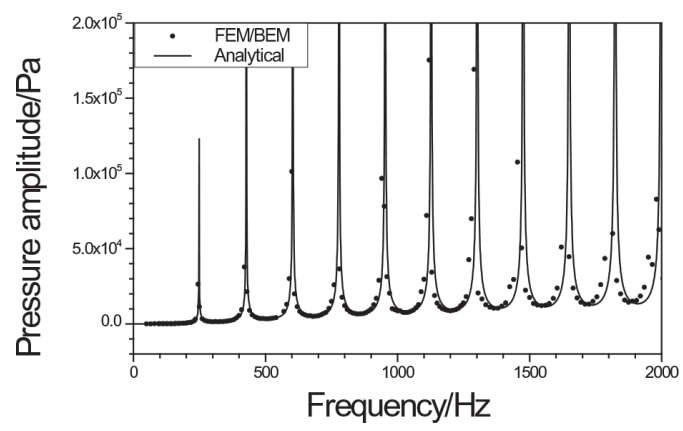

Figure 5: Frequency response of exterior surface acoustic pressure amplitude.

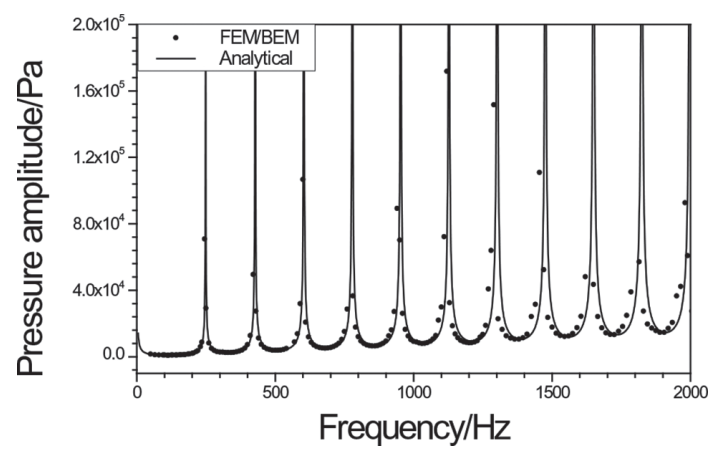

Figure 6: Frequency response of interior surface acoustic pressure amplitude. 
comparison of the interior surface acoustic pressure amplitude obtained using coupled FEM/BEM and the corresponding analytical solutions. Clearly, the numerical results agree with the corresponding analytical solutions.

\section{CONCLUSIONS}

A coupled finite element method and boundary element method are developed to model sound transmission through thin elastic shell with different fluids on the inside and outside of the shell. Burton \& Miller's composite formulation is employed to avoid the nonuniqueness problem existing in the exterior acoustic boundary element method. To validate the present method, sound transmission through an air-filled and submerged in sea water spherical shell is simulated.

\section{ACKNOWLEDGEMENT}

This project was supported by NSFC (11172132) and NSFC (51405225); the Priority Academic Program Development of Jiangsu Higher Education Institutions.

\section{REFERENCES}

[1] Junger, M.C. \& Feit, D., Sound Structure and Their Interaction, 2nd ed., MIT, Cambridge, MA, 1986.

[2] Mason, W.P. \& Thurston, R.N., Physical Acoustics, Vol. XV, London: Academic Press, INC, pp. 191-285, 1981.

[3] Ciskowski, R.D. \& Brebbia, C.A., Boundary Element Methods in Acoustics, Southampton Boston: Computational Mechanics Publications, pp. 61-74, pp. 109-129, 1991.

[4] Junger, M.C., Sound scattering by thin elastic shells. The Journal of the Acoustical Society of America, 24, pp. 366-373, 1952.

https://doi.org/10.1121/1.1906905

[5] Goodman, R.R. \& Stern, R., Reflection and transmission of sound by elastic spherical shells. The Journal of the Acoustical Society of America, 34, pp. 338-344, 1962. https://doi.org/10.1121/1.1928120

[6] Burton, A.J. \& Miller, G.F., The application of integral equation methods to the numerical solution of some exterior boundary value problems. Proceedings of the Royal Society A: Mathematical, Physical and Engineering Sciences, A323, pp. 201-210, 1971. https://doi.org/10.1098/rspa.1971.0097

[7] Luke, C.J. \& Martin, P.A., Fluid-solid interaction: acoustic scattering by a smooth elastic obstacle. SIAM Journal on Applied Mathematics, 55(4), pp. 904-922, 1995. https://doi.org/10.1137/s0036139993259027

[8] Zienkiewicz, O.C., Kelly, D.W. \& Bettess, P., The coupling of the finite element method and boundary solution procedures. International Journal for Numerical Methods in Engineering, 11, pp. 355-375, 1977.

https://doi.org/10.1002/nme.1620110210

[9] Wilton, D.T., Acoustic radiation and scattering from elastic structures. International Journal for Numerical Methods in Engineering, 13, pp. 123-138, 1978. https://doi.org/10.1002/nme.1620130109

[10] Mathews, I.C., Numerical techniques for three dimensional steady-state fluid-structure interaction. The Journal of the Acoustical Society of America, 79, pp. 1317-1325, 1986. https://doi.org/10.1121/1.393711 
[11] Wu, T.W. \& Dandapani, A., A boundary element solution for sound transmission through thin panels. Journal of Sound and Vibration, 171, pp. 145-157, 1994. https://doi.org/10.1006/jsvi.1994.1110

[12] Jeans, R.A. \& Mathews, I.C., A unique coupled boundary element/finite element method for the elastoacoustic analysis of fluid-filled thin shells. The Journal of the Acoustical Society of America, 94, pp. 3473-3479, 1993. https://doi.org/10.1121/1.407201

[13] Cook, R.D., Concepts and Applications of Finite Element Analysis, John Wiley \& Sons, Inc: New Yorks, pp. 99-118, 191-207, 1974.

[14] Yan, Z.Y., Sound Transmission Through Thin Elastic Shell with Different Fluids on Both the Inside and Outside. Ph. D Thesis, Shanghai Jiao Tong University, P.R. China, 2000.

[15] Yan, Z.Y., Hung, K.C. \& Zheng, H., Solving the hypersingular boundary integral equation in three dimensional acoustics using a regularization relationship. The Journal of the Acoustical Society of America, 113, pp. 2674-2683, 2003.

https://doi.org/10.1121/1.1560164 